\title{
Simulation of the fluorescence signal detected by a space telescope for extreme energy cosmic ray observations
}

\author{
Zohra Bouhali ${ }^{1, *}$ and Taoufik Djemil ${ }^{1,2, * *}$ \\ ${ }^{1}$ Laboratoire de Physique des Rayonnements, Badji Mokhtar University, Annaba, Algeria. \\ ${ }^{2}$ Faculté de Médecine, Badji Mokhtar University, Annaba, Algeria.
}

\begin{abstract}
The experimental technique of fluorescence light measurement is used for indirect observations of cosmic ray particles at very high energies. Extensive Air Showers (EAS) initiated by Extreme Energy Cosmic Rays (EECRs), up to $100 \mathrm{EeV}$ and entering the Earth's atmosphere, are simulated with the CORSIKA package. The influence of different simulation parameters on the EAS characteristics is studied, especially on the longitudinal distribution of charged particles, depth of shower maximum and energy released to the air. By taking the atmospheric scattering of light into account, the number of fluorescence photons, with wavelengths between $300-430 \mathrm{~nm}$, and their arrival time distribution to an ideal space telescope are calculated.
\end{abstract}

\section{Introduction}

Ultra High Energy Cosmic Rays (UHECR) are the most energetic particles observed in nature with detected energies up to $3-5.10^{20} \mathrm{eV}$. The flux of such particles reaching Earth is extremely low ( 1 particule. $\mathrm{km}^{-2}$. century ${ }^{-1}$ ). The experimental observations of UHECR are performed nowadays by the Pierre Auger Observatory (PAO) in Argentina and the Telescope Array (TA) observatory in the USA. The observation of these particles leads to many interesting questions mainly on their nature and origin. JEM-EUSO, which stands for Joint Experiment Missions for Extreme Universe Space Observatory, is a new type of observatory embarked on the ISS, and uses the Earth's atmosphere as a detector. It will observe transient luminous phenomena taking place in the atmosphere of the Earth and caused by particles coming from Space. The sensor is a super-wide field telescope that will detect primary particles with energy above $10^{20} \mathrm{eV}$.

\section{Methods}

In this work, we have calculated the number of charged particles and the energy deposit in the atmosphere for an extensive air shower initiated by a proton, iron or photon primaries entering the US standard atmosphere with energy up to $100 \mathrm{EeV}$. The ground impact of the shower axis was at the nadir of the telescope. We have used for this purpose different low and high energy hadronic interaction models available in the CORSIKA package [1]. QGSJETII-04 [2], EPOS [3] and SYBILL [4] were used to treat hadronic interactions at high energy $\left(E_{L a b}>80\right.$ $\mathrm{GeV}$ ). While below this value, GHEISHA [5] as well as
UrQMD [6] were used. The electromagnetic interactions are generated by the EGS4 [7] code system.

We have used a thinning level, $l_{t h}=10^{-4}$, and a maximum weight limitation, $w_{\max }$, to ensure high simulation quality within a reasonable computing time limit. We have taken $w_{\text {max }}^{l}=l_{t h} * E(\mathrm{GeV})$ for the electromagnetic particles and $w_{\max }^{l}=10^{-2} * l_{t h} * E(\mathrm{GeV})$ for hadronic ones. In addition to thinning algorithm, the use of the CONEX [8] program highly reduces the computation time by combining a fully MC simulation at high energy with a fast numerical cascade equation below certain threshold energies.

As a first step, we have calculated the energy released in air due to the development of an EAS with the help of the CORSIKA package. The contributions from particles falling below the energy threshold of the simulation to the energy released have been taken into account. Then, we have obtained the UV fluorescence photon yield which depends on the energy released, air density and temperature following Eq.1:

$$
F Y\left(K_{c}, \rho, T\right)=\frac{\left(\frac{d E}{d X}\right)}{\left(\frac{d E}{d X}\right)_{K_{c}}} \frac{A \times \rho}{1+B \times \rho \times \sqrt{T}}
$$

where $\frac{d E}{d X}$ is the average energy deposit of all particles in the shower, $\left(\frac{d E}{d X}\right)_{K_{c}}$ the energy deposit for an electron with kinetic energy $K_{c}=0.85 \mathrm{MeV}, \rho$ the air density and $\mathrm{T}$ the temperature. The constants $\mathrm{A}$ and $\mathrm{B}$ are calculated and listed by M. Nagano et al. [9], for 14 wave bands between $329-428 \mathrm{~nm}$.

For this work, only the attenuation due to Rayleigh scattering was taken into account. Multiple scattering of light and ozone absorption was ignored. The Atmospheric transmission $T_{R}$ is given by Eq.2:

$$
T_{R}=\exp ^{-\delta_{\text {rayl }}}
$$

\footnotetext{
*e-mail: bouhali_zohra@yahoo.fr

**e-mail: taoufik.djemil@univ-annaba.dz
} 


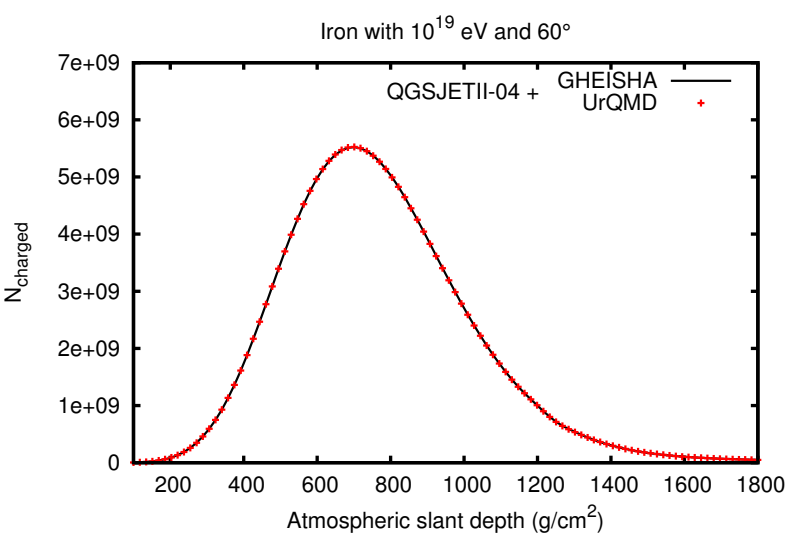

Figure 1. Number of charged particles given by QGSJETII04 alternatively combined with low energy hadronic interaction models GHEISHA and UrQMD, for an incoming primary iron with $E=10^{19} \mathrm{eV}$ and $\theta=60^{\circ}$.

were $\delta_{\text {rayl }}$ is the optical depth and for Rayleigh scattering is given by Eq.3 [10]:

$$
\begin{aligned}
\delta_{\text {rayl }}=\frac{X}{3102 \mathrm{~g} / \mathrm{cm}^{2}}\left(\frac{\lambda}{400 \mathrm{~nm}}\right)^{4} & \\
& \left(1-0.0722\left(\frac{\lambda}{400 \mathrm{~nm}}\right)^{2}\right)^{-1}
\end{aligned}
$$

where $X$ is the atmospheric slant depth along the photon path and $\lambda$ is the wavelength (in nm). Finally, the number of photons created per shower length interval and arriving to the detector pupil is given by Eq.4:

$$
N_{p h}^{d e t}=\frac{\Delta \Omega}{4 \pi} \times T_{R} \times F Y\left(K_{c}, \rho, T\right)
$$

where $\Delta \Omega$ is the detector solid angle, $F Y$ the fluorescence yield per charged particle, $T_{R}$ the atmospheric transmittance.

\section{Results}

The influence of the hadronic interaction models on the longitudinal distribution of the EAS has been studied. We have calculated the number of charged particles and energy deposit in the atmosphere for an EAS initiated by an iron primary with an energy $E=10^{19} \mathrm{eV}$ and a zenith angle $\theta=60^{\circ}$. For the low hadronic interaction model, our results illustrated by Fig. 1 and Fig. 2 show a difference less than 5\% between the GHEISHA and UrQMD models. The same results were found for incident protons. For the remaining simulation, we have then chosen the GHEISHA model because it also takes less computing time in the simulation compared to UrQMD.

For the high energy hadronic interaction model, Fig. 3 and Fig. 4, we have used three models namely QGSJETII04, EPOS and SYBILL. We have noticed a difference less than $10 \%$ between EPOS and QGSJETII-04 and less than

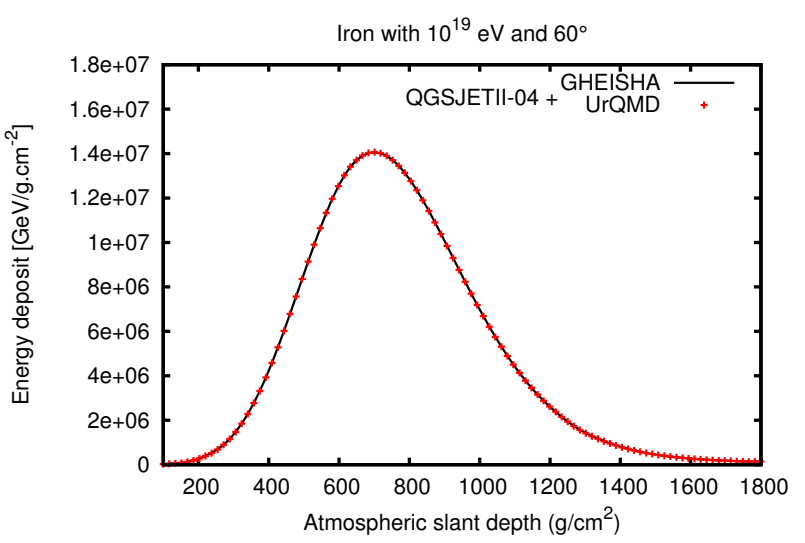

Figure 2. Energy deposit in air given by QGSJETII-04 alternatively combined with low energy hadronic interaction models GHEISHA and UrQMD, for an incoming primary iron with $E=10^{19} \mathrm{eV}$ and $\theta=60^{\circ}$.

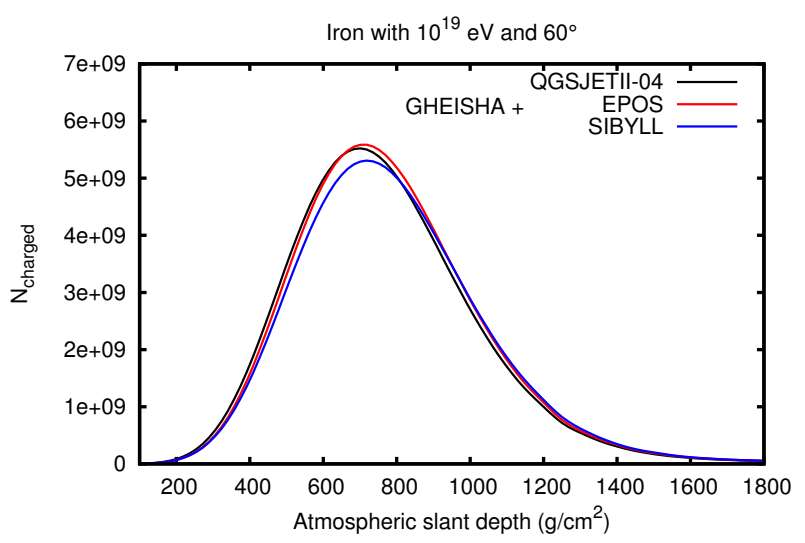

Figure 3. Number of charged particles given by GHEISHA alternatively combined with high energy hadronic interaction models QGSJETII-04, EPOS and SIBYLL, for an incoming primary iron with $E=10^{19} \mathrm{eV}$ and $\theta=60^{\circ}$.

20\% between QGSJETII-04 and SIBYLL. So we have chosen the widely used QGSJETII-04 because it takes less computation time and gives relatively close results to those obtained by EPOS.

After having selected the hadronic interaction models, QGSJETII-04 and GHEISHA, we need to validate our method. Therefore, we have compared our results to experimental measurements obtained by the $1500 \mathrm{~m}$ Array of PAO. We have computed the average deposit energy distribution of the secondary particles in the atmosphere in the same condition than those of PAO [11]. 500 events were simulated for each primary proton, iron and photon with an energy $E=3 \times 10^{19} \mathrm{eV}$ and a zenith angle $\theta=60^{\circ}$. This comparison is shown in Fig.5 and the results are consistent with the experimental data. We have also computed the depth of shower maximum $X_{\max }$ for a proton and iron with extreme energies and compared our results to those measured by the PAO [12]. This comparison is shown in 


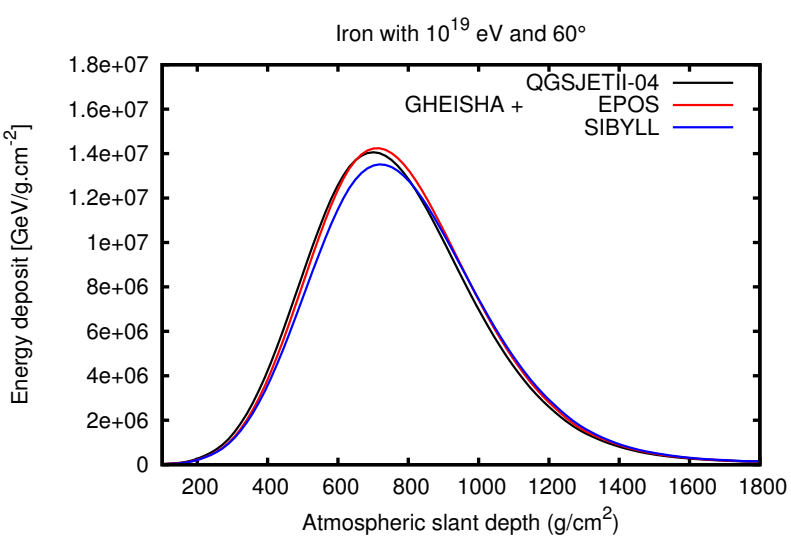

Figure 4. Energy deposit given by GHEISHA alternatively combined with high energy hadronic interaction models QGSJETII04, EPOS and SIBYLL, for an incoming primary iron with $E=10^{19} \mathrm{eV}$ and $\theta=60^{\circ}$.

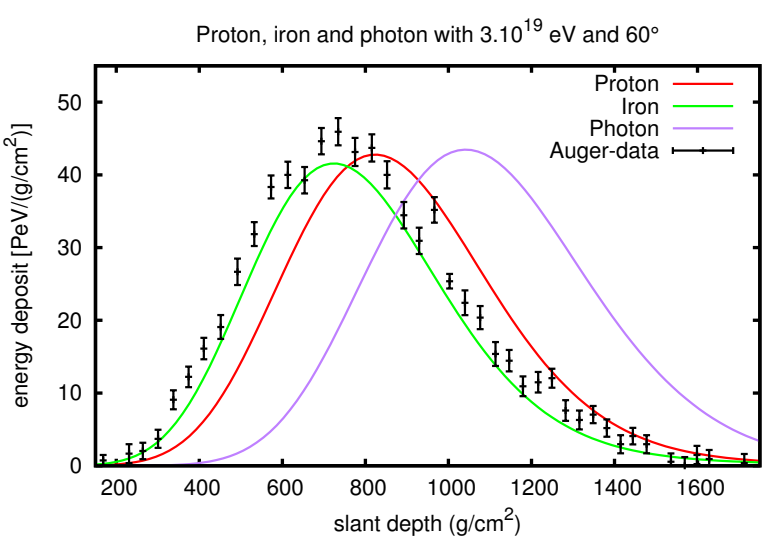

Figure 5. Profile of the average energy deposit in air for 500 EAS initiated by primary particles, proton, iron and photon, with energy $E=30 \mathrm{EeV}$ and zenith angle $\theta=60^{\circ}$. Experimental data points are taken from [11].

Fig.6 with consistency between calculations and experimental data.

After this validation, we have calculated the fluorescence signal detected by an ideal space telescope and produced by typical extreme energy cosmic ray particles. For this aim, we have simulated for each primary particle, namely proton, iron and photon, 500 events with $E=10^{20}$ $\mathrm{eV}$ and $\theta=60^{\circ}$. The number and arrival time of the fluorescence photons reaching the detector pupil of the telescope aboard the ISS have been calculated. The results are shown in Fig.7. One can see that iron produces more UV photons than proton and photon primaries. Also, its detected signal occurs sooner than the two others. These features are related to the depth of shower maximum which is lower for iron showers than for proton and photon ones. The upward photons are more transmitted when their production occurs at higher altitudes. Finally, the number of fluorescence photons detected is $7406 \pm 49,7055 \pm 96$ and $5231 \pm 153$ for iron, proton and photon primaries respec-

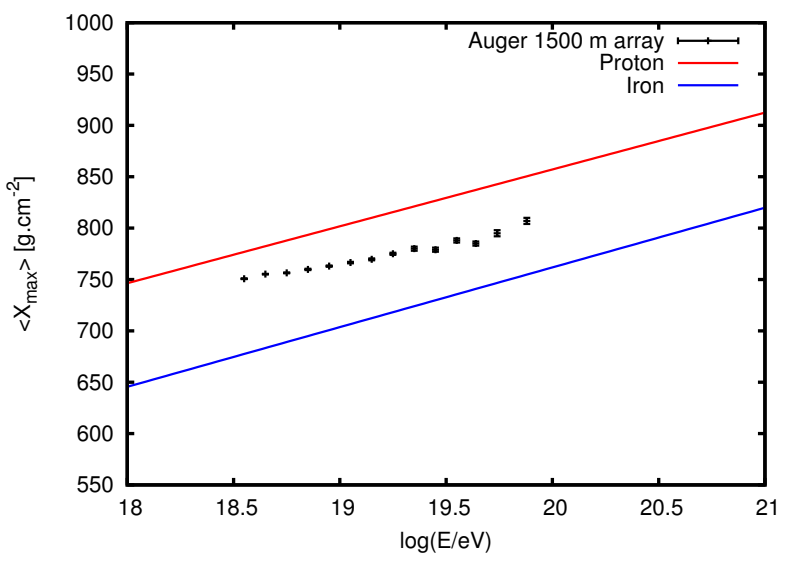

Figure 6. Distribution of the depth of shower maximum $X_{\max }$ obtained by our simulation compared to the experimantal data measured by the $1500 \mathrm{~m}$ Array of the PAO [12].

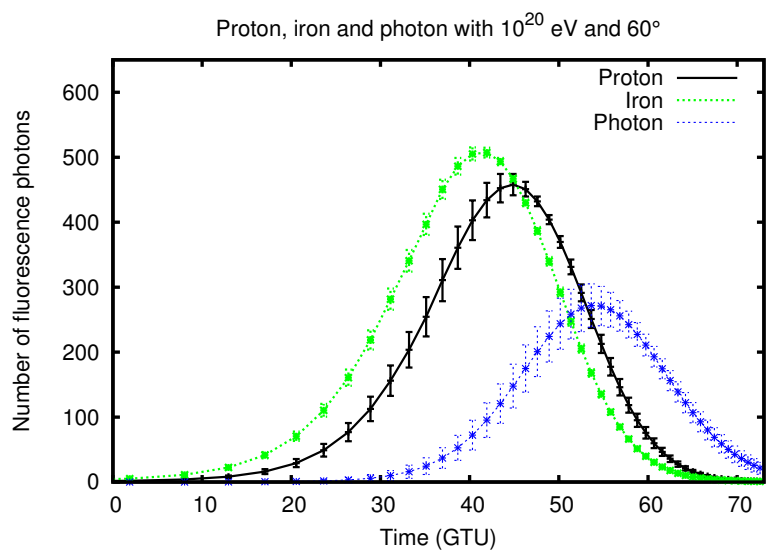

Figure 7. Average number of fluorescence photons between 329$428 \mathrm{~nm}$ recorded by an ideal detector aboard the ISS as a function of time $(1 \mathrm{GTU}=2.5 \mu \mathrm{s})$. The primary particles are proton, iron and photon with $E=100 \mathrm{EeV}$ and $\theta=60^{\circ}$. For each primary particle, 500 events were generated.

tively. This result is in a good agreement with the calculation done by M. Bertaina et al. [13], with the help of the EUSO Simulation and Analysis Framework (ESAF). In their simulation, they found that 7131 fluorescence photons will reach the detector pupil for a primary proton with $E=10^{20} \mathrm{eV}$ and $\theta=60^{\circ}$.

\section{Conclusion}

In this work, we have performed a calculation of the number of the UV fluorescence photons arriving at the detector pupil of an ideal telescope aboard the ISS. Also, their arrival time profile has been determined. With the help of the CORSIKA package, we have calculated the longitudinal energy deposit in air by EAS initiated by a typical EECR particle. Then, this deposit energy has been converted into UV fluorescence photons which are propagated to a space detector aboard the ISS. The fluorescence signal generated exhibits a posssible discrimination between 
light and heavy primaries. This can be used to identify the EECR particle entering the Earth's atmosphere but high statistics in the measurements are required.

\section{References}

[1] D. Heck, et al., Report fzka 6019 (1998).

[2] S. Ostapchenko, Phys. Rev. D 83 (2011) 014018.

[3] T. Pierog, et al., Phys. Rev. C 92 (2015) 034906.

[4] R. S. Fletcher, et al., Phys. Rev. D 50 (1994) 57105731.

[5] H. Fesefeldt, Report pitha-85/02 (1985).
[6] S. Bass, et al., Prog. Part. Nucl. Phys. 41.255 (1998) 255.

[7] W. Nelson, et al., Report slac 265 (1985).

[8] T. Pierog, et al., in: Proc. $32^{\text {nd }}$ Int. Cosmic Ray Conf., contr. 1170, Beijing (China).

[9] M. Nagano, et al., Astrop. Phys. 22 (3) (2004) 235.

[10] C. Berat, et al., Astrop. Phys. Vol.33, 4, P. 221-247 (2010)

[11] J. Abraham, et al., Nucl. Instrum. Methods Phys. Res. A 620 (2010) 227.

[12] A. Aab, et al., Physical Review D 96, 122003 (2017)

[13] M. Bertaina, et al., Advances in Space Research 53 (2014) 1515-1535. 